\title{
Comportamiento agronómico del cultivo del plátano, variedad curare enano en Sandy Bay Costa Caribe Norte de Nicaragua
}

\author{
Karen Yaskara Castellón Muller ${ }^{1}$ \\ William Benjamin Pineda ${ }^{2}$ \\ Enrique Cordón Suárez ${ }^{3}$
}

\section{Resumen}

Se evaluó el comportamiento agronómico en el cultivo del plátano (Musa paradisiaca) de la variedad curare enano en la comunidad indígena Waitna tigni, Sandy Bay Norte, Región Autónoma Costa Caribe Norte (RACCN). Es de carácter experimental, con la siembra de 150 cepas de plátano con peso promedio de 3.5 libras, en un área de $30 \mathrm{x}$ $40 \mathrm{~m}^{2}$. Se utilizó el método triangular con una distancia de 3 metros entre planta y planta e hilera.

El manejo aplicado en el cultivo fue semi tecnificado, se realizó con el método tradicional empleado por los comunitarios que toman en cuenta la época de siembra, el clima, las fases lunares, y emplean como herramientas azadones y machetes; este proceso se complementó con la aplicación de fertilizantes, fungicida y nematicida con un plan de aplicación y de manejo del cultivo.

Los resultados reflejaron que las variables número de hojas, diámetro de tallo, ahijamiento y dedo (largo, ancho y peso), se asemejan a su carta tecnológica. El rendimiento general fue de 38,250 unidades de plátano por hectárea, equivalente a 14,750 kilogramos por hectárea del producto. De manera general el cultivo presentó un buen comportamiento agronómico, fenológico y de adaptabilidad, por lo que se puede implementar un programa de producción con buen manejo y con fines de comercialización nacional e internacional.

Palabras clave: Cultivo; agronómico; variables; manejo.

\section{Summary}

The agronomic behavior of plantain (Musa paradisiaca) of the enano curare variety was evaluated in the Waitna tigni indigenous community, Sandy Bay Norte, Autonomous Regions of the North Caribbean Coast (RACCN). It has an experimental nature, with the planting of 150 plantain strains with an average weight of 3.5 pounds, in an area

\footnotetext{
Ingeniera Agroforestal. Correo: myaskara@yahoo.es

Ingeniero Agroforestal. Correo: bpineda57@yahoo.com

Doctor en Agroforestería Tropical. Vicerrector de la Universidad de las Regiones Autónomas de la Costa Caribe Nicaragüense Recinto Universitario Bilwi. Correo: encordon@yahoo.com
}

Recibido: 07/03/2017 Aprobado: 23/11/2017 
of $30 \times 40 \mathrm{~m}^{2}$. The triangular method with a distance of 3 meters between plant and plant and row was used.

The management applied in the crop was semi-technified, it was carried out with the traditional method used by the community people that take into account the sowing season, the climate, the lunar phases, and use as tools hoes and machetes; this process was complemented with the application of fertilizers, fungicide and nematicide with an application and crop management plan.

The results showed that the variables number of leaves, stem diameter, tillering and finger (length, width and weight) resemble to their technological chart. The overall yield was 38,250 units of plantain per hectare, equivalent to 14,750 kilograms per hectare of the product. In general, the crop showed a good agronomic, phenological and adaptability behavior, which is why a production program with good management can be implemented at a national and international marketing purposes.

Key Words: Crop: agronomic; variables; management.

\section{Introducción}

El plátano (Musa acuminata/Musa balbisiana) como alimento es considerado uno de los cultivos más importantes en el mundo, ocupa el cuarto lugar en importancia después del arroz, trigo y leche. Se cultiva extensivamente en los trópicos y en las zonas templadas. Es apreciado por su sabor, valor nutritivo y su disponibilidad como alimento durante todo el año. Representa una importante fuente de alimentos en áreas rurales de casi todos los países tropicales y subtropicales.

La comunidad de Sandy Bay se ubica en el litoral norte del municipio de Puerto Cabezas (a orillas del mar Caribe), posee una población de 2,500 habitantes y su sistema productivo se basa principalmente en la pesca de escamas y langosta. También practican una agricultura de pequeña escala y de subsistencia en los bosques de galerías (aledaños al río Ulang), con una baja diversificación de cultivos basada en: arroz, quequisque, yuca y plátano. Generalmente este último cultivo es el de menor producción y difícil adaptación.

En la RACCN la producción y consumo es significativa. La producción promedio es entre 4-7 toneladas por año (MAGFOR, 2010), y se obtiene principalmente de unidades productivas pequeñas y trabajadas por las familias. Generalmente la demanda de plátano en la RACCN es mayor que la oferta, por lo que se complementa este déficit con plátano traído desde el sector del Pacífico de Nicaragua.

En las comunidades indígenas miskitus el plátano es bien apetecido; empero, su producción es mínima. Según los comunitarios, de manera general este cultivo 
es de difícil adaptación ya que sufre de muchas enfermedades, siendo más crítica la producción en el sector de los litorales.

Conociendo las dificultades de producción que presenta el cultivo de plátano en las comunidades indígenas de la RACCN, específicamente en la zona de Sandy Bay, se realiza esta investigación en la comunidad de Waitna tigni, con el fin de valorar el comportamiento agronómico del cultivo de Plátano variedad Curaré Enano, a las condiciones agroecológicas de la zona.

\section{Revisión de literatura}

\section{Manejo agronómico}

Son labores culturales que se hace en un cultivo específico para mejorar la producción y rendimiento por unidad de área, también llamado: Buenas Prácticas Agrícolas (BPA) tales como: riego, aporque, despunte, resiembra, deshije, podas, decapitación floral. El manejo agronómico del cultivo de plátano consiste en realizar las buenas prácticas como la selección de semillas, el control de malezas, las prácticas culturales como el deshoje, deshije, desbellote, desmane, entre otras; como también realizar un programa de fertilización y las prevenciones de las plagas y enfermedades.

\section{Morfo fisiología}

La planta de plátano y el banano son clasificadas como monocotiledóneas; hierba estolonífera perenne, su tallo verdadero permanece corto hasta su diferenciación floral. Las hojas son grandes y oblongas, poseen pseudo peciolos largos, que se ensanchan en vainas cuya imbricación forma el falso tallo o pseudotallo. (Belalcazar, 1991).

Después de haber producido un determinado número de hojas funcionales, el meristemo central experimenta una acción hormonal que detiene la diferenciación de brotes foliares y determina el inicio de la floración. No solo se detiene la producción de hojas, sino también la producción de raíces, por lo que comienza un período verdaderamente crítico para la planta. (Agrocadena de plátano, 2008). Por otro lado, Rodríguez y Guerrero (2002), explican que el proceso de floración tiene una duración de aproximada de tres meses. El tallo floral nace en el cormo y sube a través del pseudotallo, hasta que emerge la inflorescencia.

\section{Requerimiento Edafoclimáticas}

Belalcázar (1999) afirma que según la variedad, el plátano puede cultivarse desde el nivel del mar hasta los 2000 metros de altura con temperaturas promedio entre 22 ${ }^{\circ} \mathrm{C}$ y $28^{\circ} \mathrm{C}$. Requiere de alta luminosidad y precipitación bien distribuida de $150 \mathrm{~mm}$ mensuales. Los vientos huracanados, el exceso de agua y las sequías prolongadas, son 
los peores enemigos del cultivo. Según (Marcelino et al, 2004), son preferibles las llanuras húmedas próximas al mar, resguardadas de los vientos y regables. El crecimiento se detiene a temperaturas inferiores a $18^{\circ} \mathrm{C}$, produciéndose daños a temperaturas menores de $13^{\circ} \mathrm{C}$ y mayores de $45^{\circ} \mathrm{C}$.

\section{Propagación}

Según Marcelino et al, (2010), el plátano se reproduce por medio de material vegetativo denominado colinos, cormos, cepas o hijos; es la principal vía de transmisión de las características genéticas deseables.

\section{Variedades de plátano}

La variedad que tiene mayor aceptación en los mercados de varios países es el curare enano (Lardizabal, 2007), porque tiene las mismas características organolépticas y de proceso que el cuerno; pero, con un mejor rendimiento en campo. El curare enano tiene las siguientes ventajas:

- Produce mayor cantidad de dedos para exportación después del desmane.

- Es de porte bajo y de fácil cosecha.

- El tamaño y grosor del dedo es aceptable.

- Ya desmanado, el cual se deja con 4 a 5 manos - para que el racimo quede con un mínimo de 35 dedos - se obtienen dedos que pesen por lo menos $340 \mathrm{~g}$ ( 0.75 Lb.), con un largo mínimo de $25 \mathrm{~cm}$ (10 pulgadas).

\section{Establecimiento y siembra de plátanos}

Para la siembra de plátano utilizamos material vegetativo (cormo) que debe de proceder de plantas libres de enfermedades y daño de insectos. Si no ponemos la atención debida a esta labor, se puede estar introduciendo en las áreas de siembra, picudo, nematodos, Erwinia, etc.

\section{Distancias y técnicas de siembra}

Según Velásquez V.M. (2003), la distancia recomendada depende de: tipo de mantenimiento que se le dará a la plantación, si es para mercado nacional o exportación, si se asociará con otros cultivos; la fertilidad del suelo y Variedad. Las distancias varían de 3 × 3 metros en cuadro o en triángulo, lo cual implica 1.111 y 1.280 plantas por hectáreas. Los arreglos cuadrados y triangulares son fáciles de implementar. Los arreglos de doble surco tienen dos hileras pegadas (1 a $1.5 \mathrm{~m}$ de separación) y un espacio grande ( 3 a $4 \mathrm{~m}$ entre hileras dobles); esto facilita las labores culturales y los controles fitosanitarios. 


\section{Fertilización}

Las primeras fases de crecimiento de las plantas son decisivas para el desarrollo futuro, por tanto es recomendable en el momento de la siembra utilizar un fertilizante rico en fósforo. Cuando no haya sido posible la fertilización inicial, la primera fertilización se hará cuando la planta tenga entre 3-5 semanas. Se recomienda abonar al pie que distribuir el abono por todo el terreno, ya que esta planta extiende poco las raíces.

A los dos meses aplicar urea o nitrato amónico y repetir a los 3 y 4 meses. Al quinto mes se debe hacer una aplicación de un fertilizante rico en potasio, por ser uno de los elementos más importantes para fructificación del cultivo. En plantaciones adultas, se seguirá empleando una fórmula rica en potasio, distribuida en el mayor número de aplicaciones anuales, sobre todo en suelos ácidos.

\section{Crecimiento y desarrollo}

La alta densidad de los cultivos ejerce una gran influencia sobre el crecimiento y desarrollo de cada planta debido a la competencia por luz que genera dentro de la comunidad (Cayón, 1992).

Según Marcelino et al (2004), se comercializan diferentes variedades y/o clones de plátano Los plátanos comerciales, se pueden clasificar según las características morfológicas de sus racimos, como se presenta en el siguiente cuadro:

Tabla 1: Características de las diferentes variedades de plátano

\begin{tabular}{|l|l|l|l|l|l|l|}
\hline \multirow{2}{*}{ SUBCLON } & \multicolumn{5}{|c|}{ CARACTERÍSTICAS } \\
\cline { 2 - 7 } & \multicolumn{2}{|c|}{ PLANTA } & \multicolumn{2}{c|}{ RACIMO } & \multicolumn{2}{c|}{ DEDOS } \\
\cline { 2 - 7 } & ALTURA (m) & CIRCUNFRENECIA (cm) & $\begin{array}{c}\text { PESO } \\
(\mathbf{k g})\end{array}$ & $\begin{array}{c}\text { DED0 } \\
(\text { racimo })\end{array}$ & LONGITUD (cm) & $\begin{array}{c}\text { DIÁMETRO } \\
(\mathbf{c m})\end{array}$ \\
\hline $\begin{array}{l}\text { CUERNO } \\
\text { ROSADO }\end{array}$ & $3.30-4.50$ & $48.5-55.34$ & 11.5 & 28 & 24.1 & 15.0 \\
\hline $\begin{array}{l}\text { CUERNO } \\
\text { BLANCO }\end{array}$ & $3.48-4.65$ & $50.6-59.28$ & 13.2 & 35 & 25.5 & 15.5 \\
\hline $\begin{array}{l}\text { CURARE } \\
\text { ENANO }\end{array}$ & $3.25-4$ & $55.9-66.25$ & 12.8 & 45 & 25 & 15.0 \\
\hline
\end{tabular}




\begin{tabular}{|l|l|l|l|l|l|l|}
\hline \multirow{3}{*}{ SUBCLON } & \multicolumn{5}{|c|}{ CARACTERÍSTICAS } \\
\cline { 2 - 7 } & \multicolumn{2}{|c|}{ PLANTA } & \multicolumn{2}{c|}{ RACIMO } & \multicolumn{2}{c|}{ DEDOS } \\
\cline { 2 - 7 } & ALTURA $(\mathrm{m})$ & CIRCUNFRENECIA $(\mathbf{c m})$ & $\begin{array}{c}\text { PESO } \\
(\mathbf{k g})\end{array}$ & $\begin{array}{c}\text { DEDO } \\
(\mathbf{r a c i m 0})\end{array}$ & LONGITUD $(\mathbf{c m})$ & $\begin{array}{c}\text { DIÁMETRO } \\
(\mathbf{c m})\end{array}$ \\
\hline FHIA 20 & $3.00-4.40$ & $12.3-15.0$ & 26.7 & 75 & 28.7 & 15.5 \\
\hline FHIA 21 & $3.00-4.60$ & $50.6-71.0$ & 20.9 & 75 & 28.7 & 15.5 \\
\hline
\end{tabular}

\section{Producción de plátano}

El Ministerio de Fomento (2009), en su estudio encontró que la mayor parte de la producción mundial del plátano está destinada a suplir el consumo interno de los países productores y sólo una pequeña parte es comercializada en los mercados internacionales. Robinsón y Alberts (1986) indicaron que el objetivo de la explotación de plátanos es obtener la máxima producción ha-1 año-1

La variedad de plátano con mayor aceptación en Centroamérica es el Curaré Enano el cual mantiene las mismas propiedades y características organolépticas y de proceso que otras variedades; pero posee altos rendimientos en campo, mayor cantidad de dedos después del desmane, longitud y grosor aceptable al mercado y es de fácil manejo en campo. Su porte bajo permite implementar prácticas agronómicas que mejoran su eficiencia tales como acame y riego por goteo (Lardizábal y Gutiérrez 2006).

De acuerdo a Belalcázar et al (1994), la densidad de población en plátano está condicionada por la distancia de siembra y el número de plantas por cada sitio de producción y puede influir positiva ó negativamente en los componentes de desarrollo y producción.

\section{Materiales y métodos}

Es de carácter experimental debido a que se investiga el crecimiento, desarrollo y rendimiento del cultivo de plátano Curare enano, en áreas comunales con ecosistemas de sabanas (edafología y clima), aledaños a los litorales. Desde la concepción de la idea de investigación se solicitó a las autoridades de las comunidades el permiso de ingreso al área de trabajo; también se les explicó la importancia del estudio para la seguridad alimentaria de la población de la comunidad, y los objetivos trazados. Los líderes comunales aceptaron desarrollar el proceso investigativo con el acompañamiento comunal.

El universo del estudio lo constituye la siembra de 150 cepas de plátano de variedad curaré enano en un área de $30 \times 40 \mathrm{~m}^{2}$, en una parcela comunal. El muestreo 
consistió de 30 plantas de plátano, representativas de todo el universo, así como de buen porte y estructura de planta.

El material vegetativo para la implementación de esta labor se seleccionó desde el departamento de León (Finca Santa Isabel), las cepas tenían un peso promedio de 3 a 5 libras $\left(15 \mathrm{X}_{10} \mathrm{~cm}\right)$ conteniendo yemas en buen estado.

Tabla 2: Etapa vegetativa y reproductiva del plátano

\begin{tabular}{|l|l|l|l|}
\hline \multicolumn{1}{|c|}{ Variable } & \multicolumn{1}{|c|}{ Método de medición } & \multicolumn{1}{c|}{ Frecuencia de recolección de datos } & \multicolumn{1}{c|}{ Instrumento } \\
\hline $\begin{array}{l}\text { Altura de } \\
\text { planta }\end{array}$ & $\begin{array}{l}\text { Del pseudotallo hasta la base } \\
\text { de la hoja. }\end{array}$ & Medido cada 2 meses dds. & Cinta métrica. \\
\hline $\begin{array}{l}\text { Diámetro del } \\
\text { tallo }\end{array}$ & $\begin{array}{l}\text { A mitad sobre la altura de la } \\
\text { planta. }\end{array}$ & Medido cada 2 meses dds. & Cinta métrica. \\
\hline $\begin{array}{l}\text { Número de } \\
\text { hojas }\end{array}$ & Por conteo manual & Conteo cada 2 meses dds. & Manual. \\
\hline \multicolumn{1}{|c|}{ Etapa reproductiva } & A partir de la floración de la planta. & Manual. \\
\hline $\begin{array}{l}\text { Número de } \\
\text { Hijos }\end{array}$ & Por conteo Manual. & Cinta métrica. \\
\hline $\begin{array}{l}\text { Longitud del } \\
\text { racimo }\end{array}$ & $\begin{array}{l}\text { Del raquis hasta la última parte } \\
\text { del racimo. }\end{array}$ & En la madurez fisiológica. & Conteo manual. \\
\hline $\begin{array}{l}\text { Número de } \\
\text { manos }\end{array}$ & $\begin{array}{l}\text { De la densidad poblacional por } \\
\text { planta. }\end{array}$ & Madurez fisiológica. & Conteo manual. \\
\hline $\begin{array}{l}\text { Número de } \\
\text { dedos }\end{array}$ & $\begin{array}{l}\text { De la densidad poblacional por } \\
\text { planta. }\end{array}$ & Madurez fisiológica. & \begin{tabular}{l} 
Cinta métrica. \\
\hline $\begin{array}{l}\text { Longitud de } \\
\text { dedos }\end{array}$
\end{tabular} más representativa. \\
\hline $\begin{array}{l}\text { Diámetro de } \\
\text { dedos }\end{array}$ & $\begin{array}{l}\text { Se recolecto de la segunda } \\
\text { mano. }\end{array}$ & Desarrollo fisiológico. & Cinta métrica. \\
\hline $\begin{array}{l}\text { Peso por ra- } \\
\text { cimo }\end{array}$ & $\begin{array}{l}\text { Después del corte del racimo. } \\
\text { Peso de dedo }\end{array}$ & Después del corte del racima etapa del desarrollo fisiológico. & Peso. \\
\hline
\end{tabular}




\section{Resultados y discusión}

\section{Manejo agronómico implementado en el cultivo}

\section{Preparación de material}

Antes de la siembra se desinfectó las cepas con 10occ de Vanodine y 1oocc de insecticida-nematicida VYDATE en 270 litros de agua, se colocó en sacos cebolleros se dejó sumergido por 30 minutos, luego se dejó secar por 24 horas seguidamente fueron sembradas las cepas de plátano.

\section{Siembra}

La preparación de suelo se hizo de manera manual (azadones, machetes) 15 días antes de la siembra, se removió el suelo con el fin de desinfectar las larvas vivientes. Se trabajó en una parcela demostrativa comunitaria de $30 \times 40 \mathrm{~m}^{2}$. El ahoyado se realizó de acuerdo al tamaño de las cepas $(40$ x $40 \mathrm{~cm})$.

\section{Distancia de siembra}

La siembra se realizó a una distancia de 3×3 m de manera triangular, con densidades de 1,250 cepas en una hectárea. Se tomó como referencia 150 plátanos en una parcela. Generalmente las distancias de siembras recomendadas técnicamente se basan a los objetivos del agricultor dependiendo del manejo y el área a sembrar.

\section{Fertilización}

Al momento de sembrar las cepas se aplicó una onza de fertilizante completo N-P-K (12-30-10) de manera edáfica alrededor de la cepa y enterrándola con tierra para evitar el contacto directo con la cepa, se realizó esta práctica con toda la plantación en general.

A los 15 días después de siembra nuevamente se aplicó una onza de fertilizante completo en cada planta alrededor de la planta y enterrándola con tierra para evitar que se volatilice o se pierda por escorrentía. Esta dosis se realizó cada 15 días por dos meses ( 4 aplicaciones) con el fin de que se establezca el cultivo en la zona. Luego se aplicó una onza de urea a los 2 meses después de siembra y al tercer mes se continuó la aplicación de fertilizante completo mensualmente con una dosis de 3 onza por planta hasta los días de floración. 


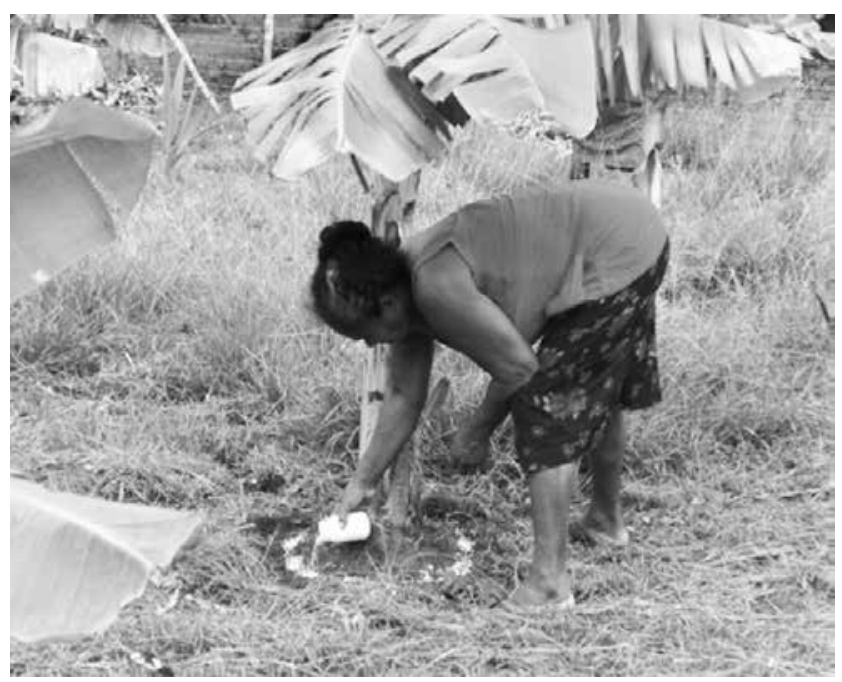

Figura 1: Aplicación de fertilizante.

Se continuó con el plan de fertilización a 3 meses después de siembra donde se aplicó 3 onza de urea a cada planta, a los 6 meses después de siembra se suministró el fertilizante foliar N-P-K (15-30-15) diluido con adherente y 0.5 libra de azúcar, con el fin de fortalecer el crecimiento foliar debido a que fue perjudicado por la sequía (2 aplicaciones, una vez por mes). También a los 8 meses después de siembra se aplicó 3 onzas de potasio con el fin de fortalecer el desarrollo de la planta, esta aplicación fue realizada hasta los días de floración.

\section{Control de maleza}

El control de maleza se realizó de la misma manera que acostumbran los comunitarios. Un primer deshierbe con machete durante las 3 primeras semanas de haberse realizado la siembra y se acompañó de un proceso de aporque de cada planta.

Antes de cada fertilización se realizaban prácticas culturales como deshoje y el control de malezas (12 y 180 dds). Se eliminaron hojas secas infuncionales y las hojas que interferían en el desarrollo normal del fruto.

\section{Control de plaga}

Primeramente se desinfectó las cepas antes de siembra con insecticida VYDATE mezclado con VANODINE, luego a los 60 días después de siembra, se aplicó el insecticida-nematicida VYDATE con 5occ diluido en 20 litros de agua de manera edáfica, realizando un chorro alrededor de la planta evitando tocar raíces, con el fin de contrarrestar alguna plaga que se encontrara en dicho sitio, la dosis fue realizada dos veces (una vez por mes). 


\section{Crecimiento y desarrollo}

\section{Altura de la planta}

Tabla 3: Comparación Datos de altura de plátano curare enano

\begin{tabular}{|l|l|l|l|l|}
\hline Días después de siembra & \multicolumn{1}{|c|}{$\begin{array}{c}\text { Altura cm } \\
\text { (Ensayo Waitna tigni) }\end{array}$} & $\begin{array}{c}\text { Carta tecnológica INTA } \\
(\mathbf{2 0 1 1})\end{array}$ & $\begin{array}{c}\text { Diámetro tallo cm } \\
\text { (Ensayo Waitna tigni) }\end{array}$ & $\begin{array}{c}\text { Carta tecnológica } \\
\text { INTA (2011) } \mathbf{c m}\end{array}$ \\
\hline 60 & 67 & 95 & 8.2 & 12.3 \\
\hline 120 & 92 & 124 & 14.7 & 17.5 \\
\hline 180 & 126 & 148 & 45.75 & 59.4 \\
\hline 240 & 158 & 220 & 50.1 & 62.0 \\
\hline 300 & 164 & 255 & 53.0 & 72.5 \\
\hline
\end{tabular}

Lardizábal y Gutiérrez 2006 revelan que la variedad de curare enano se caracteriza por su porte bajo, con una altura aproximada de $2.5 \mathrm{~m}$; no obstante la carta tecnológica del INTA refleja una altura promedio de entre 2.0 y $2.5 \mathrm{~m}$; sin embargo los resultados presentados en el cuadro de altura son de $1.64 \mathrm{~m}$ en la parcela Waitna tigni donde son aceptables debido a que se estudia el comportamiento del cultivo en dicho sitio y se concibió la producción del cultivo.

\section{Diámetro de pseudotallo}

Según Gonzales y Roque (1993), el diámetro del tallo es una variable muy importante que puede ser afectada por altas densidades de siembra, competencia por luz y agua con la elongación del tallo, favoreciendo el acame por el viento.

En nuestro ensayo, esta variable presenta un diámetro máximo a la madures fisiología de $53 \mathrm{~cm}$ de diámetro; sin embargo la carta tecnológica refleja un $72 \mathrm{~cm}$ como promedio, en los datos presentado por Marcelino et ál. 2004 presenta una circunferencia de $66 \mathrm{~cm}$. Respecto a nuestro estudio se observa una disminución del diámetro de la planta en cada una de las etapas de crecimiento del cultivo.

No obstante los resultados de nuestro estudio presentan un diámetro aceptable para el sostén de la planta y para la absorción de nutrientes.

\section{Número de hojas}

Según Rodríguez y Barrigh (1979), entre mayor cantidad de hojas por planta, mayor fotosíntesis, por tanto mayor rendimiento. 
Tabla 4: Numero de hojas por etapa de crecimiento

\begin{tabular}{|l|l|l|}
\hline Días después de siembra & $\begin{array}{c}\text { Número de hojas (Ensayo } \\
\text { Waitna tigni) }\end{array}$ & $\begin{array}{c}\text { carta tecnológica INTA } \\
(\mathbf{2 0 1 1})\end{array}$ \\
\hline 60 & 6.7 & 5.4 \\
\hline 120 & 7.9 & 10.5 \\
\hline 180 & 13.6 & 12.6 \\
\hline 240 & 13.0 & 8.9 \\
\hline 300 & 12.0 & 6.3 \\
\hline
\end{tabular}

Según Martínez 1984, señala que el plátano cuerno necesita un mínimo de 6 a 7 hojas al momento de la floración para alcanzar altos rendimientos siempre y cuando se garantice la funcionalidad de dichas hojas durante el llenado del racimo. En esta variable se tiene como promedio hasta la floración, un estimado de 12 hojas, en la carta tecnológica nos indica un máximo de 12.6.

\section{Ahijamiento por planta}

El número de hijos que una planta puede desarrollar representa un numero potencial de semillas para la siembra relacionada a su vez con el poder germinativo y propagativo del material en estudio (Mendoza, 1993).

Tabla 5: Promedio Número de hijos por planta

\begin{tabular}{|l|l|l|}
\hline $\begin{array}{c}\text { Días después de } \\
\text { siembra }\end{array}$ & $\begin{array}{c}\text { Número de hijos (Ensayo Waitna } \\
\text { tigni) 2014 }\end{array}$ & $\begin{array}{c}\text { Carta tecnológica INTA } \\
\text { (2011) }\end{array}$ \\
\hline 60 & 1.3 & 1.8 \\
\hline 120 & 2.6 & 2.9 \\
\hline 180 & 2.8 & 2.8 \\
\hline 240 & 3.5 & 3.3 \\
\hline 300 & 3.8 & 3.7 \\
\hline
\end{tabular}

El estudio en la comunidad de Waitna tigni presenta una buena producción de ahijamiento con un promedio de 3.8 retoños por planta. Al comparar con los resultados de la carta tecnológica, no existen diferencias significativas en cuanto a la producción de ahijamiento. Dependiendo de la variedad las plantas de plátano en todo su ciclo puede producir de 4-6 hijos por planta. 


\section{Componentes de Rendimiento}

Tabla 6: Promedios de variables de rendimiento de cultivo de plátano

\begin{tabular}{|c|c|c|c|c|c|c|c|}
\hline \multirow[b]{2}{*}{ Ensayos } & \multicolumn{2}{|c|}{ Racimo } & \multirow[b]{2}{*}{$\mathrm{N}^{\circ}$ de mano } & \multirow[b]{2}{*}{$N^{\circ}$ de Dedo } & \multicolumn{3}{|c|}{ Dedo } \\
\hline & Longitud (cm) & $\begin{array}{l}\text { Peso } \\
\text { (libra) }\end{array}$ & & & Longitud (cm) & Circunferencia (cm) & Peso (lb) \\
\hline Waitna tigni & 47.8 & 26.2 & 6.6 & 30.6 & 24.1 & 17 & 0.57 \\
\hline $\begin{array}{l}\text { Carta tecnológi- } \\
\text { ca INTA }\end{array}$ & 67.9 & 38.8 & 7.8 & 30.7 & 24.5 & 16 & 0.62 \\
\hline
\end{tabular}

\section{Rendimiento general}

El rendimiento es el producto final de un cultivo y está relacionado con la variedad de la cepa y el manejo agronómico realizado durante el ciclo del cultivo.

\begin{tabular}{|l|l|l|}
\hline \multicolumn{1}{|c|}{ Unidades de medida } & \multicolumn{1}{c|}{ Waitna tigni } & Carta tecnológica del INTA \\
\hline Unidades por racimo & 30.6 & 30.7 \\
\hline Unidades por parcela & 4590 & - \\
\hline Unidades por Hectárea & 38,250 & 38,375 \\
\hline Kg / Plantas & 11.8 & 17.5 \\
\hline Kg / Parcela & 1,770 & - \\
\hline Kg / Hectárea & 14,750 & 21,875 \\
\hline
\end{tabular}

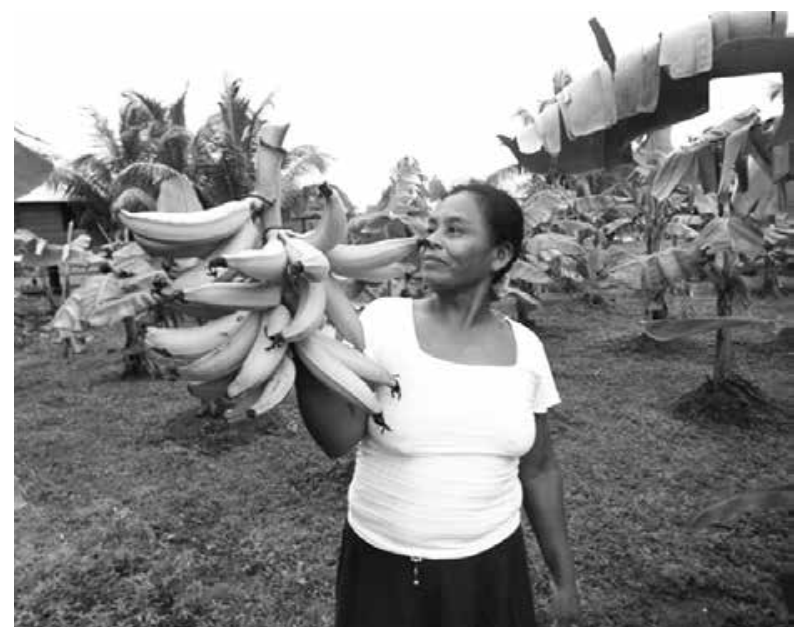

Figura 2: Producto final de un cultivo. 
Se observa que los rendimientos presentados en la comunidad de Waitna tigni son inferiores al datos en comparación; sin embargo son rendimientos aceptables para las comunidades indígenas del litoral norte considerando la producción para autoconsumo familiar.

\section{Conclusiones}

1. El ecosistema de las comunidades de Sandy Bay es de buen potencial para desarrollar el cultivo de plátano variedad Curare enano, partiendo que el cultivo se adapta fácilmente a una diversidad de zonas edafoclimáticas.

2. Los mejores resultados obtenidos lo reflejan las variables número de hojas, diámetro de tallo, ahijamiento y dedo (largo, ancho y peso), presentando datos que se asemejan a su carta tecnológica.

3. La variable racimo (longitud, peso) presentó datos un tanto menor a los referidos en la carta tecnológica; sin embargo, esto no repercutió en la producción del cultivo ya que se obtuvo un rendimiento aceptable para la comunidad.

4. El rendimiento general presentado es de 38,250 unidades de plátano por hectárea, equivalente a 14,750 kilogramos por hectárea del producto, un tanto por debajo a los referidos estudios en comparación.

5. A pesar de que el cultivo fue afectado por una pequeña sequía que duró cerca de tres meses, de manera general el cultivo presentó un buen comportamiento agronómico, fenológico y de adaptabilidad, por lo que se puede implementar un programa de producción con buen manejo y con fines de comercialización nacional e internacional.

\section{Lista de referencias}

Araya Artavia, Jorge Mario (2008). Agrocadena de plátano caracterización de la agrocadena.

Belalcázar, S. (1999). El cultivo del plátano: Guía práctica. Manizales, Colombia. Publiartes. $38 \mathrm{p}$

Belalcázar, S., J. A. Valencia y M. I. Arcila (1994). Estudio sobre densidades de población en plátano clon Dominico-Hartón (Musa AAB, Simmonds) en Colombia. p. 535-548. En: Miguel A. Contreras, José A. Guzmán, Luís R. Carrasco (Eds.). Memorias ACOBAT X Reunión de la Asociación para la Cooperación en Investigación de Bananos en el Caribe y en América Tropical (10, 1991, Tabasco, México).

Cayón, G. 1992. Fotosíntesis y productividad de cultivos. Revista Comalfi 19(2):23-21. 
Lardizábal, R. y H. Gutiérrez (2006). Manual de producción de plátano de alta densidad. USAID-RED. 38 p. (en línea). Consultado el 30 de octubre de 2009. Disponible en http://www.usaid-red.org/

Lardizabal, R. (2007). Producción de plátano de alta densidad. La Lima, Cortes. Hn. 38 p.

Marcelino, L. A. Ríos D. González V. (2004). El cultivo de plátano en Panamá. Manual de Recomendaciones Técnicas para el Cultivo Tecnificado de Plátano (Musa paradisiaca L.). Pa. $30 \mathrm{p}$.

Mendoza, S. (1993). Diagnóstico agronómico,fitosanitario y económico del cultivo del platano en diferentes niveles tecnológicos en Rivas.esis de (Universidad Nacional Agraria),Managua-Nicaragua,26p.

Robinson, J.C. y A.J. Alberts (1986). Growth and yield responses of banana (cultivar "Williams") to drip irrigation under drought and normal rainfall conditions in the sub-tropics. Scientia Horticulturae 30:187-202.

VELASQUEZ, V.M. (2003). Análisis del cultivo de plátano en el marco de la apertura comercial. Curso: La apertura comercial y las oportunidades de agronegocios. UNED. San José. 